D. THE SUN - A TYPICAL G2V STAR 


\title{
REVIEW OF ASTROPHYSICAL CONCLUSIONS FROM THE UV SOLAR SPECTRA
}

\author{
S. R. POTTASCH
}

Kapteyn Astronomical Institute, Groningen

A good deal is known about the Sun from the measurement of its spectrum. The measurement of the ultraviolet spectrum has contributed to this knowledge and I shall try to summarize the results and problems of some of these investigations.

In a general way we can divide the discussion between the continuous radiation and the line radiation, and the line radiation can further be subdivided into the absorption spectrum and the emission spectrum, which can be discussed separately not only because they originate in different regions of the atmosphere, but because the physical conditions for line formation are quite different in these different regions.

Our goal in these investigations is to determine from the observed spectrum the structure of the atmosphere, i.e. the run of temperature and density as a function of height. Further we are interested in the chemical composition of the atmosphere, and to determine whether or not it is a function of height in the atmosphere.

It is interesting to know how far these investigations have proceeded not only from the point of view of studying the Sun, but also with a view to observing stars of spectral type not too different from the Sun. Because it is possible easily to study emission from various points on the disc of the Sun, and because of the great intensity of the observable emission from the Sun which enables a very detailed study of the spectrum, we can test theories and methods for general use on the Sun, and then apply them to neighbouring spectral types.

\section{The Continuous Spectrum}

The center-to-limb measurements in the visible part of the spectrum are used to determine the temperature as a function of optical depth at the frequency observed. In principle, if the limb darkening is precisely known, one can obtain the complete run of $T$ vs. $\tau_{\lambda}$ throughout the atmosphere. In practice, the errors in the observed limb-darkening curve are such that the determination of $T$ is only valid in the regions where most of the radiation comes from, i.e. the region $2 \geqslant \tau_{\lambda} \geqslant 0.2$. For this reason it is desirable to obtain limb-darkening measurements in other spectral regions. Since as we proceed into the ultraviolet part of the spectrum the absorption coefficient begins to increase strongly, this radiation comes from much further out in the atmosphere. In this way we can build a temperature model of the solar atmosphere which is probably reasonably reliable for $\tau_{5000}>10^{-4}$. At observations made at $1500 \AA$ or less, limb darkening is no longer seen: it is now limb brightening which is observed. This indicates that we are now looking at regions above the temperature minimum of the Sun, the region where the temperature is increasing outward. 
I may point out that detailed models which are given, e.g. the Bilderberg Continuum Atmosphere rely not only on ultraviolet measurements, but infrared and visual observations as well.

There is one difficulty in present studies of the solar continuum. When measuring limb darkening at two wavelengths we obtain two functions $T\left(\tau_{\lambda}\right)$, and we consider a given position in the atmosphere, let us say where the temperature $T=5000 \mathrm{~K}$, then we have two values of $\tau_{\lambda}$ corresponding to the two wavelengths. Since the $\tau_{\lambda}$ 's differ at that point because the absorption coefficient differs with wavelength, we can obtain the ratio of the two absorption coefficients. If we extend this argument to many wavelengths we can determine the variation of the absorption coefficient as a function of wavelength at a given temperature in the atmosphere. In the visual part of the spectrum, longward of $4000 \AA$, this empirically derived absorption coefficient agrees very well with that predicted theoretically on the basis of absorption by the $\mathrm{H}^{-}$ion. Below $4000 \AA$ there has always been an indication that an additional source of opacity is present. In the ultraviolet the situation becomes more difficult to interpret.

In Figure 1, taken from Bonnet (1968), the points show the empirically determined absorption coefficient in the wavelength region from 2000 to $3000 \AA$, while the solid line is a theoretical curve. The differences amount to an order of magnitude at some

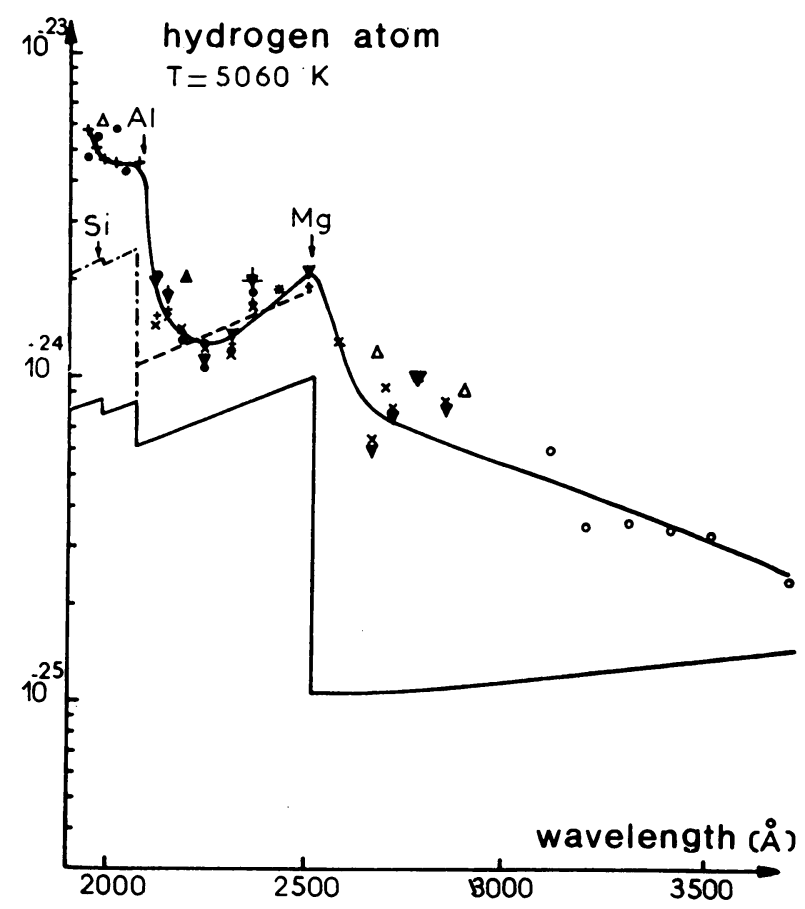

Fig. 1. A comparison of the absorption coefficient in the solar atmosphere derived from limbdarkening measures (Bonnet, 1968) and a theoretical curve. The actual absorption is seen to be as much as an order of magnitude greater than the theoretical, indicating an important source of opacity has been omitted in the theoretical calculation. Iron may be this missing source. 


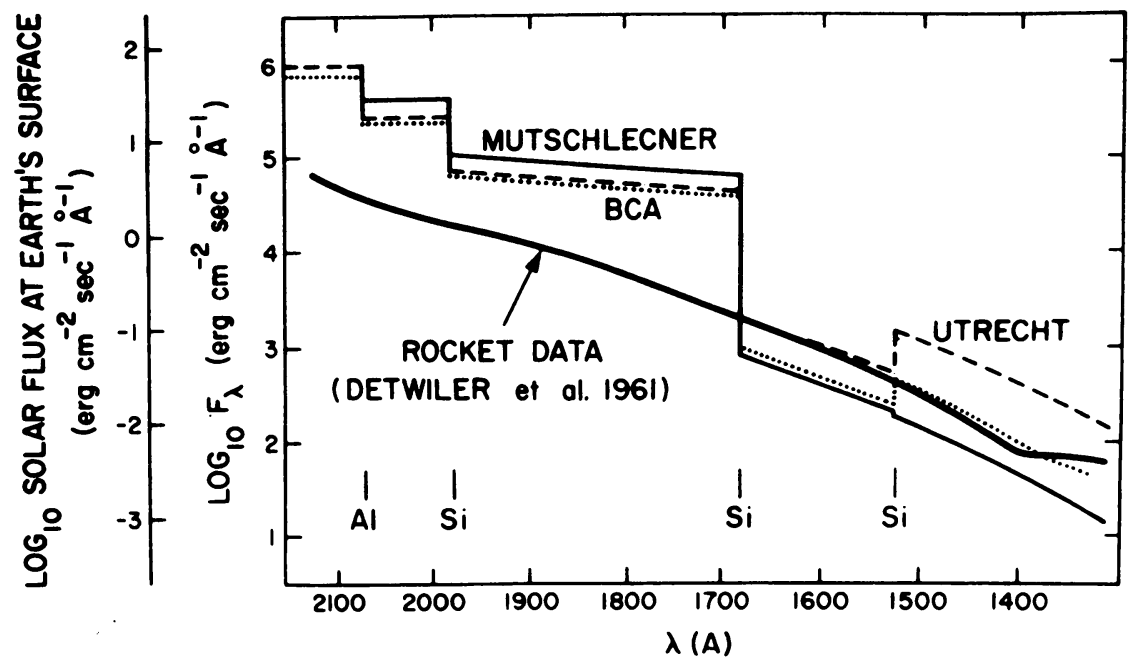

Fig. 2. A comparison of the observed flux with the theoretical prediction. The discrepancy is again due to a missing opacity source.

points, and more striking, the predicted jump at $2510 \AA$ due to $\mathrm{MgI}$, does not appear in nearly the predicted strength. This same result may be seen in a different form in the spectral region 1400-2100 $\AA$, in Figure 2, where the predicted flux is compared to observed flux. The calculations are taken from Gingerich and Rich (1968). Again differences of at least an order of magnitude appear, and a jump of intensity of almost two orders of magnitude, due to Si I, is predicted and certainly not observed in this strength. The reason for these discrepancies is that the source of the continuous absorption is poorly understood below $4000 \AA$. Probably at least a part of the continuous absorption is due to neutral iron, which has not been taken into account sufficiently, for two reasons:

(1) The absorption coefficient has not been measured experimentally and its theoretical calculation is based on a hydrogenic approximation which is known to give errors greater than an order of magnitude in silicon and magnesium.

(2) The abundance of iron has previously been underestimated by a factor of about 10-20. This brings the iron absorption in most of this spectral range to values at least as high and probably higher than silicon and magnesium.

These are at present the most important problems in the ultraviolet continuous solar spectrum.

\section{The Absorption Line Spectrum and Line Profiles}

The spectrum of the Sun above about $2000 \AA$ is an absorption line spectrum very similar to that in the visible region and the analysis also does not differ. This ultraviolet spectral region has some interest because special lines may be found here e.g. the resonance lines of singly ionized iron, and lines of certain elements which are not 


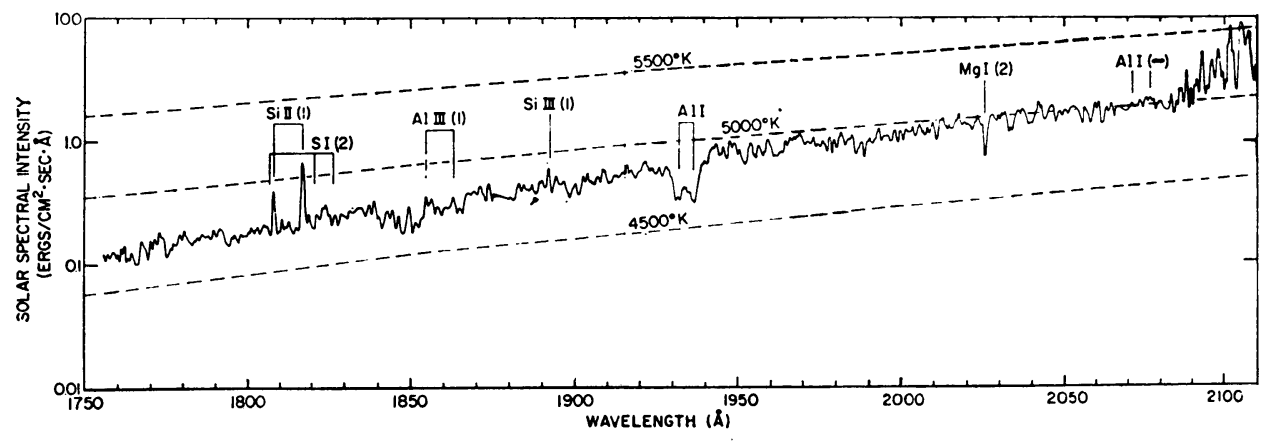

Fig. 3. The observed solar spectrum (1750-2100 $\AA)$.

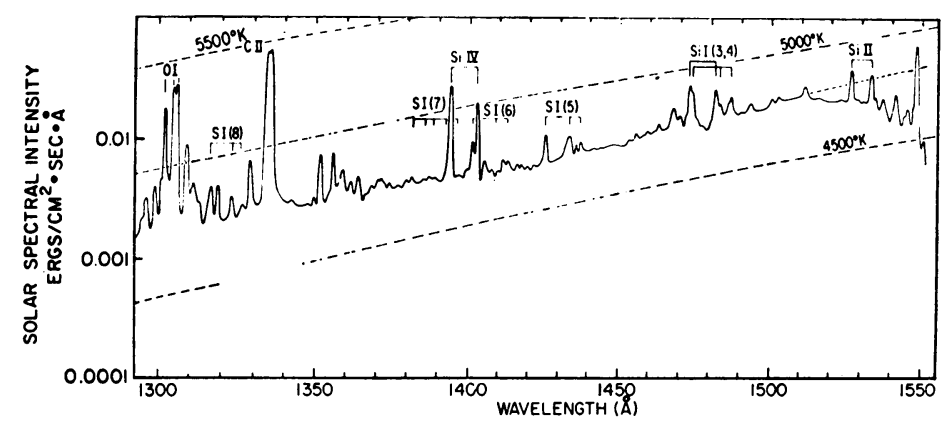

Fig. 4. The observed solar spectrum (1300-1550 $)$.

observed in the visual region are found in the ultraviolet. At present the spectrophotometry in this spectral region is of rather poor quality so that quantitative discussions are difficult.

A very special case is the MgII pair near $2800 \AA$, which have been well studied and for which high-resolution observations are available. These lines, which give similar information to the CaII lines and the Ly- $\alpha$ line, are being studied theoretically at present. These studies are not so far advanced for $\mathrm{MgII}_{\text {as }}$ for CaII, but may eventually reinforce and supplement the latter work. The difficulty at present is that knowledge of the temperature structure in the upper chromosphere where these lines are formed, is almost completely lacking.

\section{The Emission Line Spectrum of the Quiet Sun}

Below $2000 \AA$ the character of the solar spectrum changes rather suddenly from absorption line to emission line. This can easily be seen from Figures 3, 4 and 5, which illustrate the spectrum between $2100 \AA$ down to $900 \AA$. The appearance of emission means that the source function is increasing in the lines relative to the continuum as one goes to higher layers in the atmosphere. This in turn occurs through a combination of factors: 

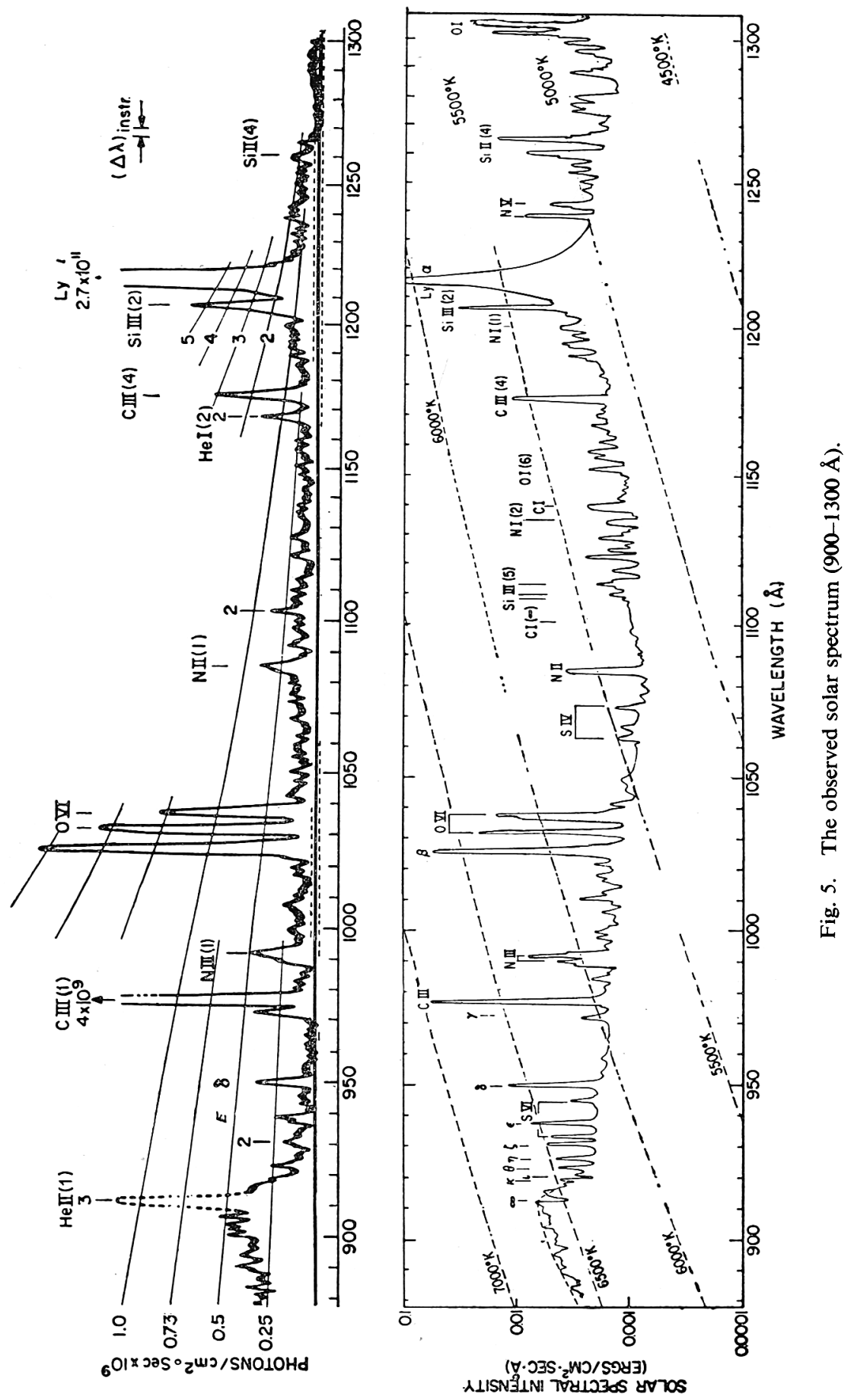
(1) The electron temperature is increasing outward, so that collisions are rapid enough to populate the high excited levels;

(2) The continuum optical depth becomes so small that the higher layers contribute only a small fraction of the continuous radiation.

Lines of SiII are observed near $1800 \AA$ and the state of ionization increases as we proceed further into the ultraviolet, as shown by Table $I$.

\section{TABLE I}

$\begin{array}{rc}\begin{array}{c}\text { Wavelength } \\ \text { interval }(\AA)\end{array} & \begin{array}{l}\text { Average ionization } \\ \text { potential }\end{array} \\ & \\ 1000-2000 & 30 \mathrm{eV} \\ 600-1000 & 60 \mathrm{eV} \\ 300-600 & 150 \mathrm{eV} \\ 40-300 & 300 \mathrm{eV} \\ 15-40 & 600 \mathrm{eV}\end{array}$

The reason for this correlation is simply that most of the observed lines are resonance lines and the wavelength of the resonance line of a particular ion is roughly correlated with the ionization potential of that ion.

I shall discuss in a summary way the analysis of the emission line spectrum before I give the results, because this kind of analysis probably is directly applicable to many low temperature stars with coronae. Only the total intensity of the line need be observed, not the line profile. The key to this analysis is the following. Since in the chromosphere and corona, where these lines are formed, we cannot assume local thermodynamic equilibrium, we must know the process of formation of these lines. Of the possible contributing processes, direct collisional excitation of the emitting level is by far the most likely since temperatures up to several million degrees are present. In comparison, radiative recombinations, which have the same density dependence, are much slower. And absorption of radiation to higher levels followed by cascading is not an important process simply because there are very few photons in the far ultraviolet continuum. If this is true the energy emitted over the surface of the Sun is

$$
I(\text { line }) \propto \int n_{L} n_{e} C_{L U}(T) \mathrm{d} v,
$$

where $n_{L}$ is the ground state population of the emitting ion (almost all observed lines are resonance lines or at least have the ground state as lower level). The collisional excitation rate $n_{e} C_{L U}(T)$ is written so that the part dependent on the electron density, $n_{e}$, is separate from the temperature-dependent part. The integration is taken over all heights in the atmosphere, or if we consider an area greater than $1 \mathrm{~cm}^{2}$, then the integral is taken over the entire volume.

Since the electrons are almost all due to the ionization of hydrogen, $n_{e} / n_{\mathrm{H}} \approx 1$. Since the ionization is all collisional and the recombination all radiative, and both 
of these rates have the same density dependence, the state of ionization depends only on the temperature, thus

Thus

$$
\frac{n_{L}}{n_{\text {element }}} \approx \frac{n_{\text {ion }}}{n_{\text {element }}}=g(T) .
$$

$$
\begin{aligned}
& I(\text { line }) \propto \int \frac{n_{\text {ion }}}{n_{\text {element }}} \times \frac{n_{\text {element }}}{n_{H}} \frac{n_{H}}{n_{e}} n_{e}^{2} C_{L U}(T) \mathrm{d} v \\
& \propto A \int n_{e}^{2} f(T) \mathrm{d} v,
\end{aligned}
$$

where we have written the abundance $n_{\text {element }} / n_{\mathrm{H}}$ as $A$, and assumed that it remains constant in the atmosphere.

The function $f(T)$ can be computed for every ion considered. It is near zero for most of the temperature range and has an appreciable value only for a limited range of temperature. We make the approximation that it has a constant value for this limited temperature range (let us say, between $T_{1}$ and $T_{2}$ ), and is zero outside of this range. The physical reason for it being zero at certain temperatures is simply either that the stage of ionization is not present or that the collisional excitation rate is very small. The equation for the intensity then becomes

$$
I(\text { line }) \propto A \int_{V\left(T_{1}\right)}^{V\left(T_{2}\right)} n_{e}^{2} \mathrm{~d} v,
$$

where the integral is taken over all the material between the temperatures $T_{1}$ and $T_{2}$. One can thus see that for every ion whose intensity is observed, we can obtain a quantity which depends on the abundance of that element and on the structure of the atmosphere where the observed line is formed.

We can separate these quantities in the following manner. Suppose we make a plot of the above observed quantity

$$
A \int_{V\left(T_{1}\right)}^{V\left(T_{2}\right)} n_{e}^{2} \mathrm{~d} v
$$

against the temperature $T_{1}$ and $T_{2}$ (or a mean value of $T_{1}$ and $T_{2}$ ) for each observed ion. An example of such a plot is shown in Figure 6 for many ions of sulphur and silicon. Notice on the diagram that curves drawn through either the silicon or sulphur ions are similar, but displaced. Since the integral which depends only on the structure of the atmosphere cannot be systematically different for the silicon and sulphur ions, the difference can only be explained by an abundance difference, i.e. silicon is about 3 times as abundant as sulphur.

This type of analysis can be extended to most of the elements observed in the ultraviolet spectrum. Only for those ions for which optical depth effects are important, 


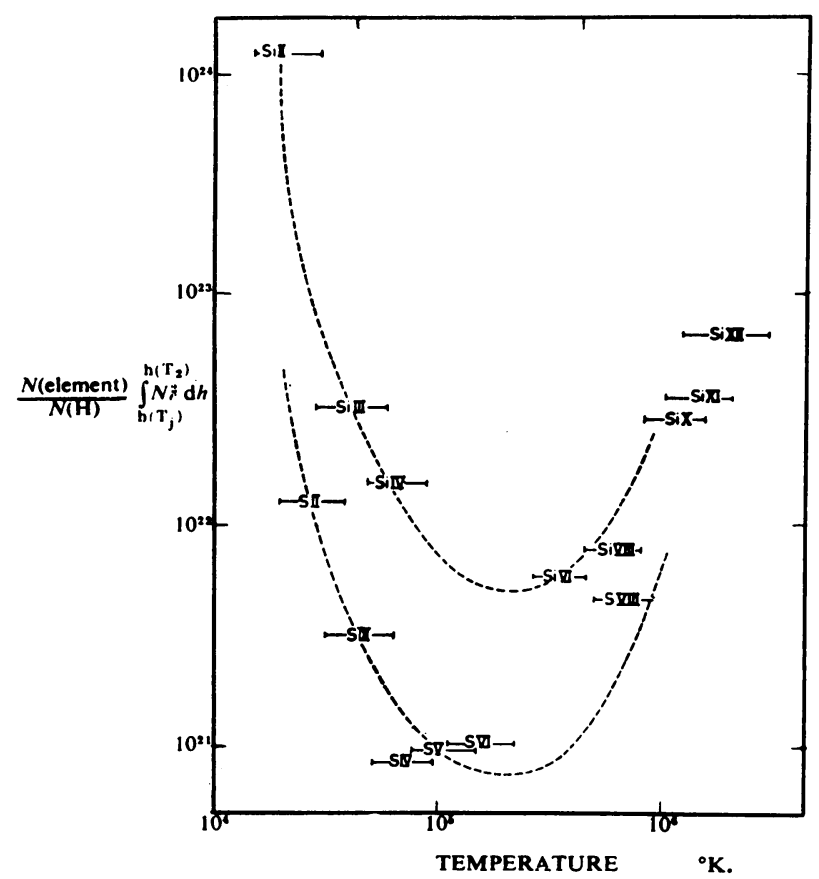

Fig. 6. The value of $N($ element $) / N(\mathrm{H}) \int_{R} N_{e}^{2} \mathrm{~d} h$ plotted against the temperature of the region of line formation for silicon and sulphur ions.

e.g. the hydrogen lines and HeI, is this method inapplicable. Thus the abundances relative to hydrogen cannot be determined solely from the ultraviolet data. Nevertheless interesting information about the abundances has been obtained in this way, e.g. that the abundances of silicon, iron and nitrogen are approximately equal in the outer solar atmosphere. More detailed abundance results are in the literature (e.g. Pottasch, 1967).

Once the abundances are known we are able to determine the value of the integral

$$
\int_{V\left(T_{1}\right)}^{V\left(T_{2}\right)} n_{e}^{2} \mathrm{~d} v,
$$

which in principle describes the distribution of density and temperature in the atmosphere. There is not enough information, however, in the integral to be able to specify the density and temperature throughout the chromosphere-corona, where the ultraviolet lines are formed. This is true even if one assumes that the atmosphere is 'homogeneous', i.e. that the density decreases, and the temperature increases monotonically with height. In the case of a nearby homogeneous atmosphere one is able to conclude that the temperature gradient must be very steep between a temperature of 60000 and $400000 \mathrm{~K}$, and these temperatures probably occur at densities several times $10^{9} \mathrm{~cm}^{-3}$.

If one makes an additional assumption about the outer atmosphere, e.g. that it is 
in hydrostatic equilibrium, one may then use the values of the integral to obtain a model of the temperature and density. Such solutions, while not unreasonable, are really not convincing, and what are really required to settle this important question are center-to-limb measurements in the ultraviolet, with sufficient angular resolution. These measurements are important not only for their direct application to the determination of the structure of the solar atmosphere, but as a guide to the interpretation of these measurements in stars, where center-to-limb observations are impossible.

\section{Ultraviolet Emission from Active Regions}

As one goes to shorter wavelengths, the emission from active regions begins to dominate the spectrum. Below 10-15 $\AA$ the active Sun gives many times the emission of the quiet Sun. It is probably mostly in the form of line emission. At $2 \AA$, lines of the heliumlike Fexxv are observed. This indicates that for short periods of time temperatures of the order of $10-30 \times 10^{6} \mathrm{~K}$ are reached. The theoretical study of these regions is just beginning and will be greatly aided by ultraviolet and X-ray observations.

It may be that some stars have more important active regions than the Sun. This probably will be more obvious in the ultraviolet spectrum than in the visible.

\section{References}

Bonnet, R. M.: 1968, Ann. Astrophys. 31, 597.

Gingerich, O. and Rich, J. C.: 1968, Solar Phys. 3, 82.

Pottasch, S. R.: 1967, Bull. Astron. Inst. Netherl. 19, 113.

\section{Discussion}

Swings: Is there no remaining problem on chromospheric iron abundance after your paper with Pecker where you used 'old' gf values?

Pottasch: The 'new' gf values of Garz and Koch for FeI will change the neutral iron abundance, but most of the iron is still in the form of singly ionized iron, so the total iron abundance which Pecker and I have found, will not be affected.

Gingerich: Although the solar model I described yesterday was based explicitly on continuum intensities at the center of the disk, its construction was guided by a knowledge of the limb darkening, especially in the critical wavelength regions where the center-to-limb variations change from darkening to brightening. The model satisfies the continuum limb-darkening observations more successfully than any other model I have seen not only in infrared and ultraviolet, but also in the visual regions.

Pottasch: The importance of your method is that, unlike a model built up entirely of limb-darkening observations, it is applicable to stars other than the Sun. For spectral types similar to the sun, an initial model may be chosen by reference to the solar model. Although in the case of stars there is no check from the limb darkening observations, criteria may be determined from a complete study of the Sun as to how successful the model is. 